\title{
Graft polymers prepared by catalytic chain transfer polymerization (CCTP) and applied as solvent-borne dispersants for carbon black dispersions of high solid
}

\author{
Yongqiang Wang, ${ }^{1}$ Guangpeng Zeng, ${ }^{2}$ Tairong Sun, ${ }^{2}$ Xinlin Hong, ${ }^{12 \star}$ Jianxiong Ling, ${ }^{2}$ \\ Gaoyong Zhang ${ }^{1}$ \\ ${ }^{1 *}$ College of Chemistry and Molecular Science, Wuhan University, Wuhan 430072 , \\ China; fax: +86-27-68756619; e-mail: hongxl@whu.edu.cn \\ ${ }^{2}$ Daoqum (Zhongshan) Chemical Enterprise Ltd., Zhongshan 528403, China
}

(Received: 02 February, 2009; published: 27 October, 2010)

\begin{abstract}
In this article, a series of graft polymer dispersants were conveniently prepared by the grafting through method, among which the macromonomer was synthesized with one-pot through CCTP technology. Rheology and transmission electron microscopy (TEM) were utilized to study the influence of amide content on the carbon black (CB) dispersing ability of these polymers in xylene. When the tertiary amino groups on the backbone were partly quaternized, the apparent viscosity of the obtained $C B$ suspensions decreased remarkably and the flocculation of the $\mathrm{CB}$ particles are effectively suppressed. TEM observations showed that the CB particles were well dispersed by the quaternized dispersant. It could be concluded that the graft polymers with a certain amount of quaternized amino groups (20 wt.\%) are an efficient dispersants of CB in organic solvent.
\end{abstract}

\section{Introduction}

Because of its excellent performance, carbon black is always thought as the most important black pigment or filler, and widely used in the industry of coatings, inks, rubber, plastics, and so forth. It is difficult to formulate stable dispersions of CB in solvent, either for pigment purposes or as reinforcing filler particles for polymers. Compared to other nanoparticles to be embedded, the primary particle size of $\mathrm{CB}$ is rather small, the interparticle forces are very high, so that aggregates and agglomerates are easily formed, which in turn weakens the application properties [1, 2]. Different kinds of dispersant such as block or graft copolymers, have been developed to solve the aggregation problem of CB application [3-11]. The necessary characteristic of an excellent polymer dispersant is that there should be anchoring functional groups and proper steric repulsive block existed in the polymer structure [12-15].

In the past decades, the industrial or scientific applications of block polymer dispersants have been studied thoroughly [16-23]. However, graft polymer dispersant has attracted little attention compared to block ones these years, even though they has been utilized widely in industries [24-26]. This may be because the preparation of the block polymer is more convenient than that of the graft one, especially after the emerging of the reversible addition-fragmentation chain transfer (RAFT) and atom transfer radical polymerization (ATRP) technologies $[27,28]$. On the other hand, functional monomers having amide group were often introduced in the dispersant structure to ensure strong anchoring to the surface of dispersed particles [29]. S. 
Creutz and $R$. Jérôme found that the amide units in block polymers, poly[2(dimethylamino) ethyl methacrylate]-b-poly(sodium methacrylate) (PDMAEMA- bPNaMA) and poly(4-vinylpyridine)-b-poly(sodium methacrylate) (4VP-b-PNaMA), were responsible for the anchoring of the polymer to titanium dioxide particles in aqueous medium $[14,15]$. Z. Wang etc. reported that poly(ethylene oxide)-b- poly[2(dimethylamino)ethyl methacrylate] (PEO-b-PDMAEMA) could anchor to the carbon nanotubes (CNT) due to the interactions between the amide units and the carboxyl groups on the surface of CNT [30]. Y.Y. Won also found that the polyisobutylene succinimide modified poly(alkylene amine) could be adsorbed onto the CB particles due to the anchoring of the poly(alkylene amine) segments [31].

In the present work, we synthesized a series of graft polymer dispersants [the trunk chain composition is Styrene (St)/butyl acrylate (BA) /2-(dimethylamino)ethyl methacrylate (DMAEMA), and the lateral chain is composed of methyl methacrylate (MMA)/butyl methacrylate (BMA)] by grafting through or macromonomer method, of which the macromonomer with a terminal active $\mathrm{C}=\mathrm{C}$ was prepared one-pot using CCTP technology. Rheological measurement and transmission electron microscopy (TEM) were utilized to study the influence of amide content on the CB dispersing ability of these polymers in xylene, before and after being quaternized.

\section{Results and discussion}

\section{Polymerization characterization}

The macromonomer is copolymerized from methacrylate monomers by the CCTP method. The structure of macromonomer with an active $\mathrm{C}=\mathrm{C}$ is confirmed by ${ }^{1} \mathrm{H}$ NMR (Figure 1), and the vinyl signal is consistent with the literature [32].
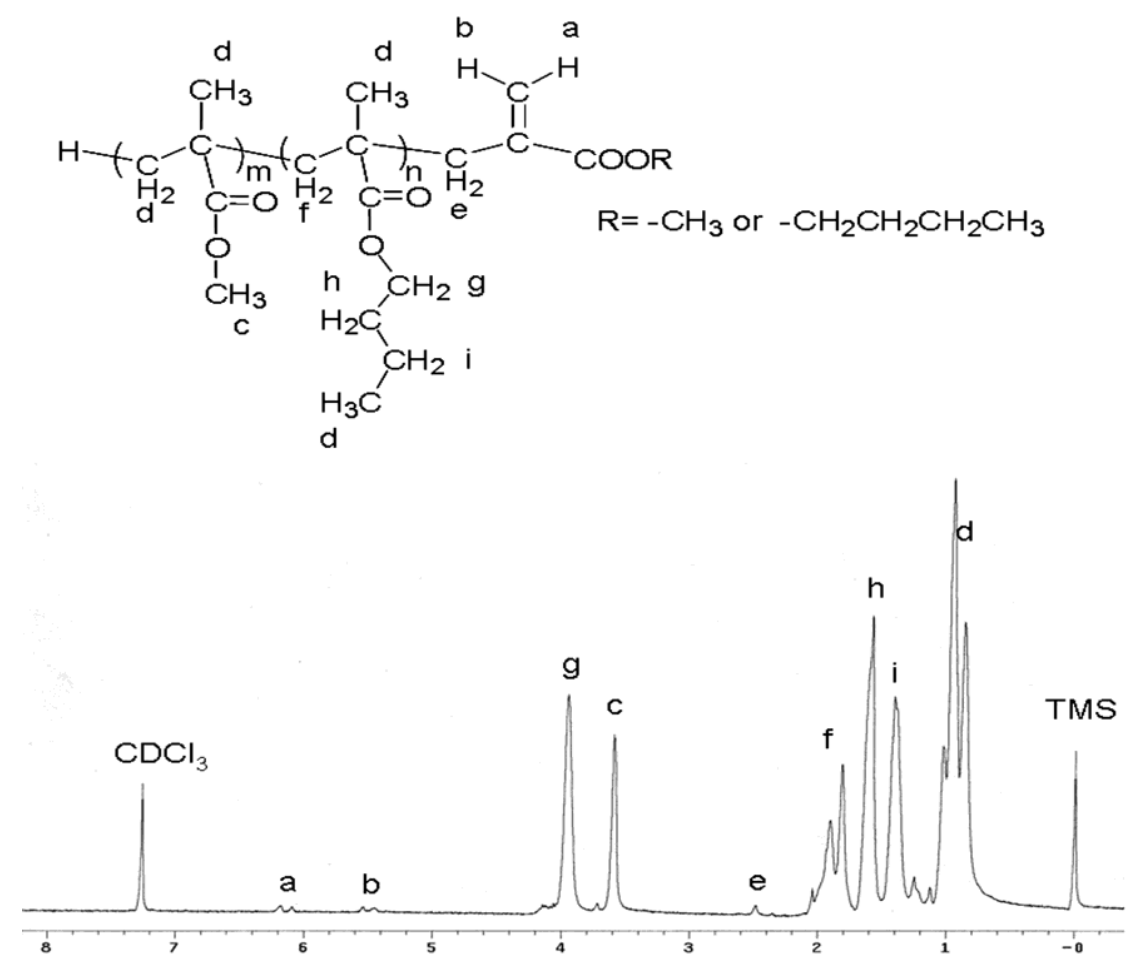

Fig. 1. ${ }^{1} \mathrm{H}$ NMR spectra of macromonomer, using $\mathrm{CDCl}_{3}$ as the solvent and TMS as the internal standard. 
The number average molecular weight and polydispersity of the polymers were determined by GPC. As shown in Table 1, the molecular weight of macromonomer is $7000 \mathrm{~g} / \mathrm{mol}$, but the number average molecular weight of the obtained graft polymer is no more than $14000 \mathrm{~g} / \mathrm{mol}$. So it could be inferred that only one macromonomer is copolymerized into the backbone in average. Based on the molecular weight of the polymer and the polymerization method, we can infer that the graft polymer is in a $\mathrm{T}$ or $L$ structure.

The structure of the graft polymers is described in Figure 2.

Tab. 1. Characteristics of the macromonomer and graft polymers.

\begin{tabular}{cccccc}
\hline & Macromonomer & $\mathrm{G}-1^{\mathrm{c}}$ & $\mathrm{G}-2^{\mathrm{c}}$ & $\mathrm{G}-3^{\mathrm{c}}$ & ${\mathrm{G}-4^{\mathrm{c}}}^{\mathrm{c}}$ \\
\hline $\mathrm{M}_{\mathrm{n}}{ }^{\mathrm{a}}$ & 7000 & 10970 & 13270 & 8950 & 9880 \\
$\mathrm{PDI}^{\mathrm{a}}$ & 1.66 & 1.58 & 1.43 & 1.42 & 1.30 \\
${\text { Conversion }(\%)^{\mathrm{b}}}^{\mathrm{b}}$ & 91 & 90 & 92 & 93 & 95
\end{tabular}

a The number average molecular weight $(\mathrm{Mn})$ and polydispersity (PDI) of the polymers was determined by GPC.

${ }^{\mathrm{b}}$ The conversion of the monomers was measured by the gravimetric method.

${ }^{c}$ The backbone compositions of the four polymers G-1, G-2, G-3 and G-4 is 85/5/10 (BA/St/DMAEMA in weight), $75 / 5 / 20,55 / 5 / 40$ and $35 / 5 / 60$ respectively. After $80 \%$ (in mole) of the amino functional groups on the backbone of the polymers are quaternized, the four graft polymers $\mathrm{G}-1, \mathrm{G}-2, \mathrm{G}-3$ and G-4 are named for QG-1, QG-2, QG-3 and QG-4 in sequence.

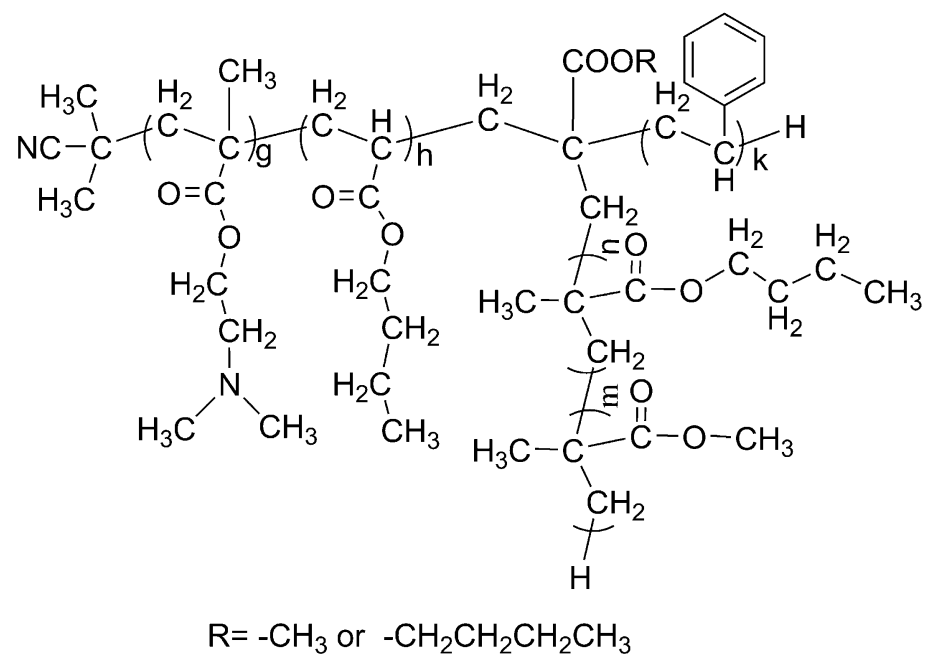

Fig. 2. The structure of the graft polymer dispersant.

The effect of the amide content on $C B$ dispersions

Figure 3a shows the flow curves of the $\mathrm{CB}$ dispersions stabilized by the synthesized graft polymer dispersants (G-1, G-2, G-3, G-4). From the curves, we found that all of the obtained dispersions are not Newtonian and thixotropic hysteresis loops existed in their flow curves. This indicates that the CB particles are not well dispersed by these unquaternized dispersants. Furthermore, with the amide content of the dispersants increased, the hysteresis loop area of the dispersions enlarges. The result shows that the flocculation occurred in the CB dispersions increases with the addition of the amide content of the dispersants. 

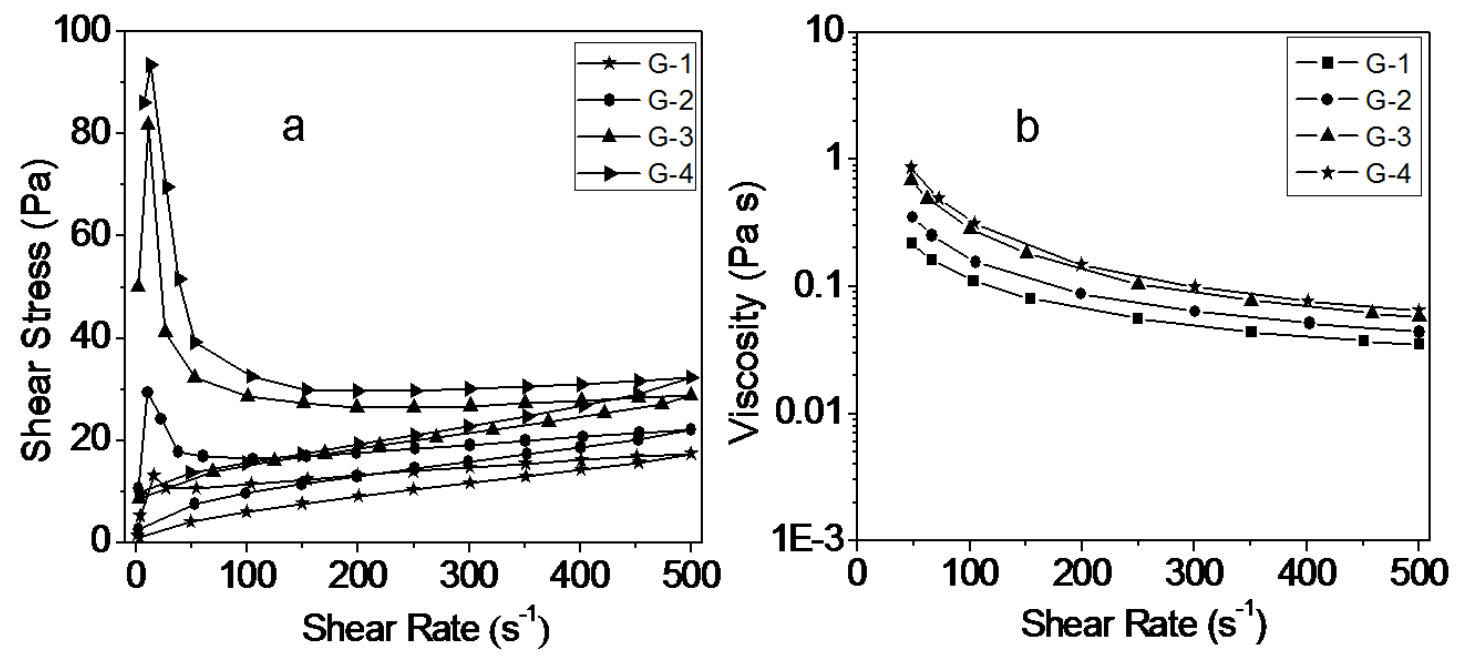

Fig. 3. The effects of the graft polymer dispersants (6wt.\%) with different content of amide unit on the rheological behavior of $20 \mathrm{wt} . \%$ CB dispersions: (a) the shear stress and (b) the apparent viscosity.

At the same time, we can also find that there is a typical peak emerging in the flow curve at the initial stage when shear rate increased, which represents the shear induced formation and destroy of the weakly flocculates in the dispersion. Since all of the rheological measurements were carried out in the same setup conditions, the shear stress value at the peak should reflect the relative flocculation intensity of the dispersions, and they are listed in Table 2.

Tab. 2. The summarized rheological results of $C B$ suspensions prepared by graft polymer dispersants.

\begin{tabular}{llll}
\hline Samples & DMAEMA content (wt.\%) & $\sigma_{\text {peak }}{ }^{\mathrm{a}}(\mathrm{Pa})$ & $\eta{ }_{100}{ }^{\mathrm{b}}$ (mPa.s) \\
\hline G-1 & 10 & 13 & 111 \\
G-2 & 20 & 29 & 156 \\
G-3 & 40 & 82 & 285 \\
G-4 & 60 & 94 & 314 \\
QG-1 & 10 & Not detected & 13 \\
QG-2 & 20 & Not detected & 8 \\
QG-3 & 40 & Not detected & 12 \\
QG-4 & 60 & 94 & 590 \\
${ }^{\mathrm{a}}$ The shear stress of the peak in flow curve. & & \\
${ }^{\mathrm{b}}$ The viscosity at the shear rate of $100 \mathrm{~s}^{-1}$. & &
\end{tabular}

From these data, the same conclusion can be drawn that more the amide content in the polymer structure, the worse dispersing ability of the dispersant in $\mathrm{CB}$ suspensions in xylene. This can also be proved by the apparent viscosity changes of these CB dispersions (Figure 3b, Table 2). The reason is due to the fact that there is no strong interaction between the dispersant backbone and the $\mathrm{CB}$ surface, and weak anchoring of the dispersant is not favorable for the dispersing of $C B$ in xylene. Besides, the amide unit is somewhat hydrophilic while the CB surface is hydrophobic, 
so the more the amide content, the worse is adsorption behavior for the dispersants to the surface of CB particles. In this viewpoint, we can conclude that high amide content in the polymer dispersant can weaken the CB dispersing ability of the graft polymer dispersant in xylene.

\section{Dispersion of $C B$ with quaternized graft polymer dispersants}

Because of the poor dispersing abilities of the above graft polymer dispersants, the quaternization of the amide groups was conducted to enhance the strong anchoring of the dispersant to the particle surface. The rheological measurement was also carried out and the corresponding data are shown in Figure 4 and Table 2. After quaternization, the flow curves of the CB dispersions prepared by using QG-1, QG-2 and QG-3, behave almost Newtonian. And the hysteresis loop disappears except QG-4 (Figure 4a). In addition, the peaks in the flow curves also disappear. The apparent viscosities are reduced remarkably, and keep almost constant for QG-1, QG-2 and QG-3, during the whole shear rate range. It should be noted that QG-2, of which the monomer (DMAEMA) content having amide group is $20 \mathrm{wt} \%$, shows the best dispersing ability among these dispersants.
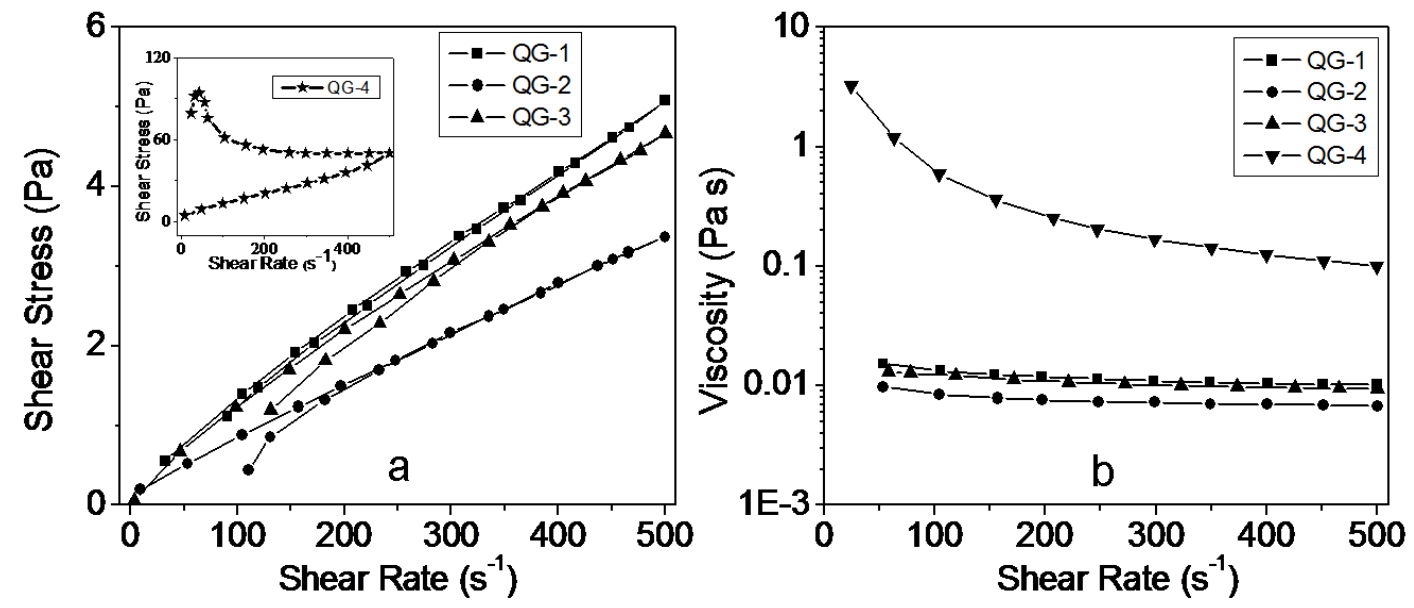

Fig. 4. The effects of the quaternized graft polymer dispersants (6wt.\%) on the rheological behavior of 20wt.\% CB dispersions: (a) the shear stress and (b) the apparent viscosity.

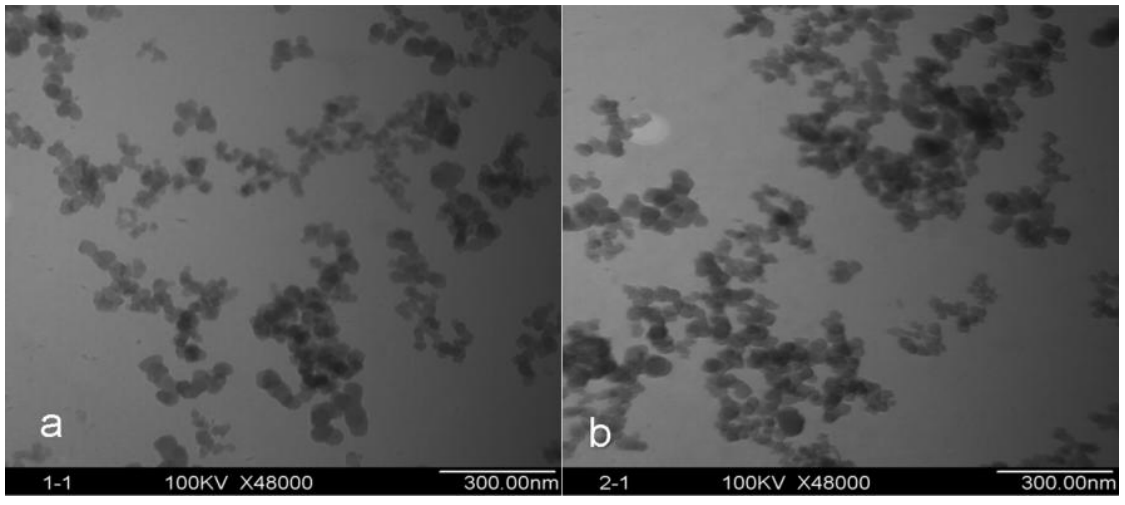

Fig. 5. TEM images of CB dispersions with 6 wt.\% of polymer dispersants (a) QG-2 and (b) G-1. 
Figure 5 is the TEM micrographs of the CB dispersions stabilized by G-1 and QG-2. At the $20 \mathrm{wt} . \%$ of $\mathrm{CB}$ content, there is much more interconnected flocculates observed in G-1 system (Figure 5b). While in QG-2 dispersion (Figure 5a), the particle size reduces remarkably, and some initial nanoparticles below $50 \mathrm{~nm}$ could be seen in TEM, even though the CB dispersion was prepared simply (ground in agate mortar for $5 \mathrm{~min}$, then dispersed by ultrasonic for $30 \mathrm{~min}$ ).

The rheological and TEM results revealed that after being quaternized properly, the adsorption of dispersant to the surface of $\mathrm{CB}$ particles can be strengthened effectively. This is because there should be two major interactions existing between dispersant and CB surface, strong ionic and weak hydrophobic interactions, according to the surface composition of CB. Known to all, most types of CB contain over 97 to $99 \%$ elemental carbon. CB may also contain chemically bound hydrogen, oxygen, nitrogen and sulfur. The oxygen content is of greatest importance for their application. Oxygen is bound to the surface in the form of mainly acidic or basic functional groups [33-35]. Thus, the quaternized anchoring chain of dispersant can provide strong ionic interaction with the surface functional groups of $\mathrm{CB}$ particles. On the other hand, absolutely most of the CB surface is hydrophobic, but the quaternized DMAEMA monomers are somewhat hydrophilic. This goes against the adsorption of dispersant to the surface of particles. So, a certain amount of quaternized groups is in favor of the polymer adsorption to the CB surface, while the adsorption may be destroyed when the amount is too large. There should be a proper balance between the ionic and hydrophobic interactions when an excellent polymer dispersant was designed to disperse CB particles in organic solvent.

\section{Conclusions}

Graft copolymers (the trunk chain composition is St/BA/DMAEMA, and the lateral chain is composed of MMA/BMA) were prepared conveniently by the 'grafting through' method, of which the macromonomer was synthesized one pot by CCTP technology. This kind of polymer was utilized as dispersant to stabilize CB particles in xylene, before and after being quaterized. The rheological and TEM investigations revealed that direct utilization of DMAEMA as anchoring monomer is not favorable to the $\mathrm{CB}$ dispersing abilities of these copolymers. Moreover, the adsorption of these dispersants to the $C B$ surface can be improved remarkably, when the DMAEMA monomer was partly quaterized. This is because the quaterized anchoring chain of dispersant can provide strong ionic interaction with the surface functional groups of $\mathrm{CB}$ particles. On the other hand, too much amount of quaterized monomers are not in favor of the adsorption of dispersant, because these hydrophilic groups would destroy the anchoring to the hydrophobic surface of CB particles. There should be a proper balance between the ionic and hydrophobic interactions for an excellent polymer dispersant to be designed to disperse CB particles in organic solvent.

\section{Experimental part}

\section{Materials}

The CB pigment in powder form was purchased from Shanghai Qiniao chemical engineering, Ltd, China, of which the specific BET surface area is $101 \mathrm{~m}^{2} / \mathrm{g}$ (measured by $\mathrm{N}_{2}$ adsorption, ASAP2020M, Micromeritics, USA). 
BMA, MMA, BA, St, DMAEMA were all purchased from Sinopharm Chemical Reagent Co., Ltd (SCRC). All monomers were purified by distillation under reduced pressure. 2, 2'-Azodisobutyronitrile (AIBN) was recrystallized from ethanol. The other reagents were all analytical grade and purchased from SCRC. The tetra-phenyl cobaloxime boron fluoride (COPhBF) as the chain transfer reagent for the CCTP was prepared according to the similar method $[36,37]$ and the structure is shown in Figure 6.

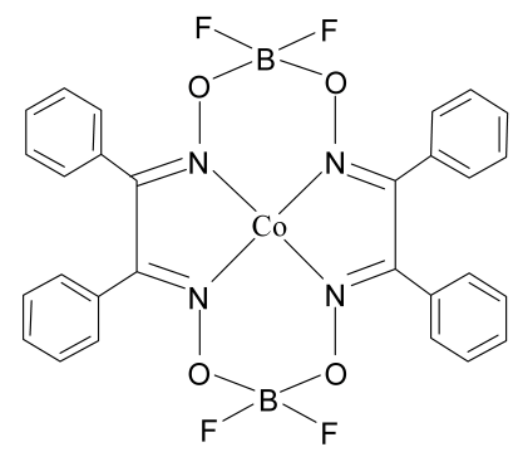

Fig. 6. The structure of COPhBF.

\section{Preparation of the graft polymer dispersants}

The macromonomer was acquired by copolymerization of the MMA and BMA via the CCTP method [38]. Briefly, $100 \mathrm{~g}$ MMA, $100 \mathrm{~g}$ BMA, $100 \mathrm{~g}$ toluene and $100 \mathrm{~g}$ butyl acetate were charged into a three-necked flask. The mixture was bubbled with high pure nitrogen (99.999\%). After bubbling for $1.5 \mathrm{~h}, 6 \mathrm{mg} \mathrm{COPhBF}$ and $1 \mathrm{~g}$ AIBN were added to the mixture and the mixture was agitated for the dissolution of the COPhBF and AIBN. Then the mixture was refluxed at $75^{\circ} \mathrm{C}$ for $6 \mathrm{~h}$. After the polymerization, the solvent and residue monomers were distilled out by rotary evaporation, and a slightly yellow solid was obtained.

Subsequently, $30 \mathrm{~g}$ macromonomer, $30 \mathrm{~g}$ backbone monomers (including BA, St and DMAEMA), $0.6 \mathrm{~g}$ AlBN, $30 \mathrm{~g}$ toluene and $30 \mathrm{~g}$ butyl acetate was added to a flask. The mixture was copolymerized at $75^{\circ} \mathrm{C}$ for $6 \mathrm{~h}$ to prepare the graft polymers [38], then the solvent and residue monomers were distilled out by rotary evaporation and the obtained solid was dried in vacuum. Based on the different compositions of the backbone monomers (weight percent) in the graft polymers, the polymers are named for G-1 (BA/St/DMAEMA=85/5/10), G-2 (BA/St/DMAEMA=75/5/20), G-3 (BA/St/DMA $E M A=55 / 5 / 40)$ and $G-4(B A / S t / D M A E M A=35 / 5 / 60)$ in the sequence of increasing of the amide content on the backbone of the polymers. After the polymerization, $80 \%$ (in mole) of the DMAEMA units on the backbone of the polymer were transferred to quaternary ammonium salt by adding amounts of benzyl chloride to the system. The quaternization reaction was operated at $90{ }^{\circ} \mathrm{C}$ for $12 \mathrm{~h}$ and it should be achieved completely under this condition because benzyl chloride is reactive. After the quaternization, the graft polymers of G-1, G-2, G-3 and G-4 are named as QG-1, QG-2, QG-3 and QG-4 in sequence.

\section{Dispersion of $C B$ in xylene}

Briefly, polymer dispersants were dissolved in xylene first, then the polymer solution was transferred to agate mortar, and CB particles were added to the polymer solution 
keeping content of CB at $20 \mathrm{wt} . \%$. The mixture was carefully ground for 5 min by the same operator and further dispersed by ultrasonic for $30 \mathrm{~min}$ before characterization.

\section{Characterization}

The number average molecular weights $\left(\mathrm{M}_{\mathrm{n}}\right)$ and polydispersity (PDI) of the synthesized polymers were measured on a gel permeation chromatography (GPC) system equipped with a Waters 515 pump, MZ gel SDplus linear $5 \mu \mathrm{m}$ column and $500 \AA$ column, Wyatt Technology Optilab DSP and DAWN ${ }^{\circledR}$ EOS $^{\text {TM }}$. THF was used as the eluent at a flow rate of $1.0 \mathrm{ml} \mathrm{min}{ }^{-1}$, and operated at $35^{\circ} \mathrm{C}$. The calibration was carried out with polystyrene standards.

${ }^{1} \mathrm{H}$ nuclear magnetic resonance ( ${ }^{1} \mathrm{H}$ NMR) spectroscopy was measured with a Varian Mercury VX-300 MHz instrument, using $\mathrm{CDCl}_{3}$ as the solvent and tetramethylsilane (TMS) as the internal standard. The TEM analysis was performed with JEM-100CXII operating at $100 \mathrm{kV}$. The rheological experiments were carried out in a rotational controlled-stress rheometer (Rheostress 600, HAAKE) in plate-plate configuration at $25^{\circ} \mathrm{C}$.

\section{Acknowledgements}

The authors gratefully acknowledge the financial support of the China National Science Foundation (NSFC-20433060), Chanxueyan Cooperative Projects of Guangdong Province and Ministry of Education (2007B090400076) and Daoqum Chemical Enterprise Ltd.

\section{References}

[1] Aoki, Y. Colloids Surf. A 2007, 308, 79.

[2] Kawaguchi, M.; Okuno, M.; Kato, T. Langmuir 2001, 17, 6041.

[3] Braun, D.; Sauerwein, R.; Hellmann, G. P. Macromol. Symp. 2001,163, 59.

[4] Miano, F.; Bailey, A.; Luckham, P. F.; Tadros, T. F. Colloids and Surf. 1992, 68, 9.

[5] Schaller, C.; Dirnberger, K.; Schauer, T.; Eisenbach, C. D. Macromol. Symp. 2002,

187, 695.

[6] Nsib, F.; Ayed, N.; Chevalier, Y. Prog. Org. Coat. 2006, 55, 303.

[7] Reuter, E.; Silber, S.; Psiorz, C. Prog. Org. Coat. 1999, 37, 161.

[8] Lin, Y.; Smith, T. W.; Alexandridis, P. Langmuir 2002, 18, 6147.

[9] Lin, Y.; Alexandridis, P. J. Phys. Chem. B 2002, 106, 10834.

[10] Ridaoui, H.; Jada, A.; Vidal, L.; Donnet, J. B. Colloids Surf. A 2006, 278, 149.

[11] Bulychev, N. A.; Arutunov, I. A.; Zubov, V. P.; Verdonck, B.; Zhang, T.; Goethals,

E. J.; Du Prez, F. E. Macromol. Chem. Phys. 2004, 205, 2457.

[12] van den Haak, H. J. W.; Krutzer, L. L. M. Prog. Org. Coat. 2001, 43, 56.

[13] Tadros, T. Adv. Colloid Interface Sci. 2003, 104, 191.

[14] Creutz, S.; Jérôme, R. Prog. Org. Coat. 2000, 40, 21.

[15] Creutz, S.; Jérôme, R. Langmuir 1999, 15, 7145.

[16] White, B.; Banerjee, S.; O'Brien, S.; Turro, N. J.; Herman, I. P. J. Phys. Chem. C 2007, 111, 13684.

[17] Nsib, F.; Ayed, N.; Chevalier, Y. Dyes and Pigments 2007, 74, 133.

[18] Zhao, L.; Gao, L. Colloids Surf. A 2003, 224, 127.

[19] Sluzarenko, N.; Heurtefeu, B.; Maugey, M.; Zakri, C.; Poulin, P.; Lecommandoux, S. Carbon 2006, 44, 3207. 
[20] Cho, J.; Daniel, I. M. Scripta Materialia 2008, 58, 533.

[21] Shin, H.; Min, B. G.; Jeong, W.; Park, C. Macromol. Rapid Commun. 2005, 26, 1451.

[22] Auschra, C.; Eckstein, E.; Mühlebach, A.; Zink, M. O.; Rime, F. Prog. Org. Coat. 2002, 45, 83.

[23] Schaller, C.; Schoger, A.; Dirnberger, K.; Schauer, T.; Eisenbach, C. D. Macromol. Symp. 2002, 179, 173.

[24] Bouhamed, H.; Boufi, S.; Magnin, A. J. Colloid Interface Sci. 2007, 312, 279.

[25] Liang, W.; Bognolo, G.; Tadros, T. F. Langmuir 2000, 16, 1306.

[26] Liang, W.; Bognolo, G.; Tadros, T. F. Langmuir 1995, 11, 2899.

[27] Moad, G.; Rizzardo, E.; Thang, S. H. Acc. Chem. Res. 2008, 41, 1133.

[28] Matyjaszewski, K.; Xia, J. Chem. Rev. 2001, 101, 2921.

[29] Spinelli, H. J. Adv. Mater. 1998, 10, 1215.

[30] Wang, Z.; Liu, Q.; Zhu, H.; Liu, H.; Chen, Y.; Yang, M. Carbon 2007, 45, 285.

[31] Won, Y. Y.; Meeker, S. P.; Trappe, V.; Weitz, D. A.; Diggs, N. Z.; Emert, J. I. Langmuir 2005, 21, 924.

[32] Suddaby, K. G.; Haddleton, D. M.; Hastings, J. J.; Richards, S. N.; O’Donnell, J. P. Macromolecules 1996, 29, 8083.

[33] Smith, D. M.; Chughtai, A. R. Colloids Surf. A 1995, 105, 47.

[34] Bradley, R. H.; Sutherland, I.; Sheng, E. J. Colloid Interface Sci. 1996, 179, 561.

[35] Lopez-Ramon, M. V.; Stoeckli, F.; Moreno-Castilla, C.; Carrasco-Marin, F. Carbon 1999, 37, 1215.

[36] Heuts, J. P. A.; Kukulj, D.; Forster, D. J.; Davis, T. P. Macromolecules 1998, 31, 2894.

[37] Bakaĉ, A.; Brynildson, M. E.; Espenson, J. H. Inorg. Chem. 1986, 25, 4108.

[38] Hu, Z.; Wang, Y.; Zeng, G.; Zhang, C.; Xia, B.; Hong, X.; Zhang, G. Acta Chimica Sinica 2007, 65, 2656. 